\title{
FISIOLOGIA DA ATIVIDADE MOTORA
}

\author{
Carlos Eduardo NEGRÃO* \\ Cláudia Lúcia de Moraes FORJAZ**
}

\section{INTRODUÇÃO}

O Laboratório de Fisiologia da Atividade Motora possui, no momento, uma única linha de pesquisa intitulada "Efeitos agudo e crônico do exercício físico sobre o sistema cardiovascular" A importância do estudo do sistema cardiovascular reside no fato das doenças desse sistema serem, hoje, a maior causa de morbimortalidade nos países desenvolvidos e em desenvolvimento, incluindo 0 Brasil (Brasil. Ministério da Saúde, 1988), o que leva à necessidade da investigação dos fatores que podem influir de forma positiva ou negativa na saúde cardiovascular. A atividade física tem se mostrado como um importante fator de proteção cardiovascular, porém a relação entre atividade física e função cardiovascular ainda não está totalmente esclarecida. Dessa forma, a preocupação atual de nosso laboratório é investigar essa relação, estudando:

$>$ os efcitos da atividade física sobre 0 funcionamento do sistema cardiovascular;

$>$ as características do exercício que amplificam seu efeito sobre a função cardiovascular;

$>$ a interação entre o exercício e outros fatores;

$>$ os mecanismos fisiológicos responsáveis por esses efeitos.
Para compreender essa relação de forma mais profunda, nosso laboratório tem desenvolvido pesquisas básicas e aplicadas, empregando, como modelos experimentais voluntários, humanos saudáveis e com patologias cardiovasculares específicas, e modelos animais adequados ao estudo cardiovascular. A escolha do modelo está sempre vinculada ao objetivo de cada estudo, bem como às características das técnicas experimentais que deverão ser empregadas.

Dessa forma, nosso laboratório tem se preocupado em investigar a relação entre 0 exercício físico e a função cardiovascular da forma mais abrangente e profunda possível, empregando para isso todas as técnicas e modelos experimentais disponíveis e necessários.

\section{EFEITOS AGUDOS DO EXERCÍCIO}

Dentro desse tópico, o grande ponto de investigação de nosso Laboratório tem sido a compreensão do fenômeno denominado "Hipotensão Pós-Exercício" Desde a década de 80 , estudos têm demonstrado que após uma única sessão de exercício, os níveis pressóricos, tanto sistólicos quanto diastólicos, permanecem inferiores àqueles medidos pré-exercício ou

\footnotetext{
Coordenador do Laboratório de Fisiologia da Atividade Motora da Escola de Educação Física e Esporte da Universidade de São Paulo; Unidade de Reabilitação Cardiovascular e Fisiologia do Exercício do Instituto do Coração (InCor) do Hospital das Clínicas da Faculdade de Medicina da Universidade de São Paulo.

** Assistente de Coordenação do Laboratório de Fisiologia da Atividade Motora da Escola de Educação Física e Esporte da Universidade de São Paulo.
} 
mesmo àqueles medidos em um dia controle sem a execução de exercícios físicos, o que tem sido denominado "Hipotensão Pós-Exercício" (Kenney \& Seals, 1993).

Apesar da existência da hipotensão pós-exercício estar bem documentada na literatura, suas características, os fatores que influem em sua magnitude e duração, e os mecanismos que a causam ainda precisam ser investigados, o que tem sido realizado por nosso Laboratório.

Em nossos estudos, temos observado que a hipotensão pós-exercício pode ser observada após uma única sessão de $\mathbf{4 5}$ minutos de exercício físico em $50 \% \mathrm{VO}_{2 \max } \mathrm{em}$ indivíduos normotensos jovens (Forjaz, Ramires, Tinucci, Ortega, Salomão, Ignês, Wajchenberg, Negrão \& Mion Junior, 1999b), indivíduos hipertensos idosos (Rondon, 1999) e ratos hipertensos (Silva, Brum, Negrão \& Krieger, 1997), mas não foi observada em indivíduos jovens hipertensos (Forjaz, Mion Junior \& Negrão, 1999a), indivíduos idosos normotensos (Rondon, 1999) e ratos normotensos (Silva et alii, 1997). Quando tentamos verificar se essa resposta hipotensora se refletia numa redução da média pressórica nas $\mathbf{2 4}$ horas, observamos que essa sessão de exercício reduziu os níveis pressóricos de 24 horas em idosos hipertensos (Rondon, 1999) e jovens normotensos (Forjaz, Mion Junior \& Negrão, 1995), mas não nos jovens hipertensos (Forjaz et alii, 1999a) e idosos normotensos (Rondon, 1999). A análise conjunta desses dados sugere que a resposta hipotensora para uma determinada sessão de exercício depende da população estudada, o que pode explicar parcialmente alguns resultados controversos obtidos na literatura.

A partir desses dados iniciais, passamos a investigar os efeitos das características do exercício (duração e intensidade) sobre a hipotensão pós-exercício em indivíduos jovens normotensos. Quanto à duração, comparamos duas durações (25 e 45 minutos) de exercício em $50 \% \mathrm{VO}_{2 \max }$ e observamos que a queda pressórica pós-exercício foi mais precoce, maior e mais duradoura após a sessão de exercício mais longa (Forjaz, Santaella, Rezende, Barretto \& Negrão, 1998b). Por outro lado, quando comparamos o efeito de diferentes intensidades de exercício ( 30 , 50 e $80 \%$ do $\mathrm{VO}_{2 \text { max }}$ ) com duração semelhante (45 min), não observamos diferença significante na resposta hipotensora pós-exercício provocada pelas diferentes intensidades (Forjaz, Matsudaira, Rodriguês, Nunes \& Negrão, 1998a).

Uma outra questão controversa é o mecanismo hemodinâmico sistêmico responsável por essa queda pressórica pós-exercício (Kenney \& Seals, 1993). Em nosso grupo, pudemos observar que, em indivíduos idosos hipertensos (Rondon, 1999) e ratos hipertensos (Véras-Silva, Mattos, Ida, Negrão \& Krieger, 1995), a queda pressórica pós-exercício se deve à redução do débito cardíaco, devido à queda do volume sistólico. Entretanto, outros autores (Coats, Conway, Isea, Pannarale, Sleight \& Somers, 1989) têm verificado que a queda pressórica pós-exercício se deve à redução da resistência vascular periférica. Além da população estudada, a intensidade do exercício pode ser um dos fatores responsáveis pela discrepância dos resultados. Dessa forma, estamos desenvolvendo, no momento, um estudo comparando o efeito da intensidade do exercício no mecanismo hemodinâmico sistêmico responsável pela hipotensão pós-exercício, em jovens normotensos. Os dados, até o momento, sugerem que, nessa população, contrariamente à população idosa, a redução pressórica se faz por uma diminuição da resistência vascular periférica (Forjaz, Sabino, Squella, Paulo, Tinucci, Rondon, Rondon \& Negrão, 1999c).

Além dos estudos acima, tentando analisar de forma mais profunda os mecanismo responsáveis pela hipotensão pós-exercício em indivíduos jovens normotensos, estudamos o efeito do exercício sobre a atividade nervosa simpática, 0 fluxo sangüíneo do antebraço e a pressão arterial no período de recuperação de uma sessão de 45 minutos de exercício em $50 \% \mathrm{VO}_{2 \max }$ numa situação basal e durante a infusão de insulina (Forjaz et alii, 1999b). Nesse estudo, verificamos que 0 exercício reduz a pressão arterial por diminuir a atividade nervosa simpática muscular $\mathrm{e}$ aumentar o fluxo sangüineo, o que está coerente com a redução da resistência vascular periférica. Além disso, nesse estudo, apesar da infusão de insulina aumentar mais a atividade simpática após o exercício que na sessão controle, a resposta do fluxo sangüíneo e da pressão arterial a essa infusão não se modificaram, sugerindo uma menor resposta vasoconstrictora à ativação simpática após o exercício.

Dessa forma, os dados obtidos até o momento demonstram a existência do fenômeno 
da hipotensão pós-exercício, porém sua magnitude, duração, e os mecanismos responsáveis parecem depender da interação entre as características da população estudada e do exercício realizado.

\section{EFEITOS CRÔNICOS DO EXERCÍCIO}

Dentre os efeitos crônicos do exercício sobre a função cardiovascular, dois aspectos têm sido investigados em nosso Laboratório: o efeito do treinamento físico aeróbio sobre a pressão arterial e seu efeito sobre a freqüência cardíaca.

De fato, o efeito bradicárdico do treinamento físico já é bastante conhecido, porém suas características e os mecanismos responsáveis por esse efeito ainda são controversos. Em um estudo com ratos hipertensos (Gava, Véras-Silva, Negrão \& Krieger, 1995; Véras-Silva, Mattos, Gava, Brum, Negrão \& Krieger, 1997), pudemos verificar que a bradicardia de repouso póstreinamento em esteira só pode ser verificada após o treinamento de intensidade leve $(50 \%$ do $\mathrm{VO}_{2 \max }$ ), não sendo observada após o treinamento de alta intensidade $\left(85 \%\right.$ do $\left.\mathrm{VO}_{2 \max }\right)$. Nessa mesma linha de análise, estudamos, mediante do bloqueio farmacológico com atropina e propranolol, qual o mecanismo neural responsável pela redução da freqüência cardíaca póstreinamento em esteira em ratos normotensos e espontaneamente hipertensos. Pudemos observar que, nos ratos hipertensos, 0 treinamento provocava uma redução do tônus simpático, trazendo-o para o nível normalmente observado nos ratos normotensos sedentários (Gava et alii, 1995). Já nos ratos normotensos, o treinamento físico reduziu tanto o tônus simpático quanto o parassimpático, de modo que seu efeito bradicárdico não se deveu a uma modificação do balanço simpato-vagal, mas sim a uma redução da freqüência cardíaca intrínseca (Negrão, Moreira, Santos, Farah \& Krieger, 1992b). É interessante observar que, num estudo utilizando a natação em vez da esteira como forma de treinamento em ratos normotensos, observamos um aumento do tônus vagal (Gianolla, Medeiros, Kalil, Mattos, Negrão, \& Brum, 1998).
Além desses aspectos, é interessante observar que, não apenas a frequiência cardíaca de repouso, mas também a resposta taquicárdica durante o exercício de intensidade progressiva está atenuada após o treinamento físico de baixa intensidade (Negrão, Moreira, Brum, Denadai \& Krieger, 1992a), o que pode ser explicado por uma menor retirada vagal e estimulação simpática durante esse exercício.

Quanto à resposta pressórica após o treinamento físico, nossos estudos com animais têm demonstrado que 0 treinamento físico não altera os níveis pressóricos de ratos normotensos (Negrão, Irigoyen, Moreira, Brum, Freire \& Krieger, 1993), porém reduz os níveis pressóricos dos ratos espontaneamente hipertensos (VérasSilva et alii, 1997). Cabe ressaltar, no entanto, que esse efeito hipotensor só foi verificado após o treinamento de baixa intensidade $(50 \%$ do $\mathrm{VO}_{2 \text { máx }}$ ), visto que $\mathrm{o}$ treinamento de alta intensidade $\left(85 \%\right.$ do $\left.\mathrm{VO}_{2 \text { máx }}\right)$ não modificou os níveis pressóricos (Véras-Silva et alii, 1997). De forma semelhante, pudemos verificar, em indivíduos jovens, que o treinamento físico aeróbio não modifica a pressão arterial de repouso nos normotensos (Forjaz, Mion Junior \& Negrão, 1996b), mas a reduz nos hipertensos (Forjaz, Mion Junior \& Negrão, 1996a). Quando tentamos verificar, nesses indivíduos hipertensos, se esse efeito hipotensor era mantido ao longo do dia, não encontramos redução significante na pressão de 24 horas, vigília e sono (Forjaz et alii, 1996a), porém nossos voluntários, apesar de hipertensos na medida clínica da pressão arterial, apresentavam, em sua maioria, níveis normais da pressão nas 24 horas, caracterizando-se como "hipertensos do avental branco" o que pode ter influenciado nossos resultados. Assim, estamos agora continuando esse estudo, investigando indivíduos com níveis hipertensivos tanto na medida clínica quanto na medida ambulatorial da pressão arterial.

Para estudar 0 mecanismo hemodinâmico sistêmico responsável pelo efeito hipotensor do treinamento físico, estudamos em ratos hipertensos, o comportamento do débito cardíaco através da colocação de um "probe" na aorta, para medir o fluxo sangüíneo a cada batimento cardíaco, o que não poderia ser feito no homem. Por meio dessa técnica, observamos que a queda pressórica, produzida pelo treinamento físico, se deve à redução do débito cardíaco devido 
à diminuição da freqüência cardíaca, visto que o volume sistólico não se alterou com o treinamento físico (Véras-Silva et alii, 1997).

Dessa forma, os dados expostos até o momento sugerem que o treinamento físico reduz a pressão arterial da população hipertensa pela redução do débito cardíaco, que diminui devido ao efeito bradicárdico desse treinamento, o qual parece ser mediado por uma redução da atividade nervosa simpática para o coração. Por outro lado, o efeito do treinamento sobre a parte periférica do sistema cardiovascular, reduzindo a atividade nervosa simpática periférica e, conseqüentemente, a resistência vascular periférica e a pressão arterial, ainda precisa ser mais estudado.

Todos os dados apresentados até o momento, foram obtidos em condições laboratoriais, onde a maioria das variáveis intervenientes foram controladas, garantindo uma alta validade interna. Entretanto, temos em nosso Laboratório a preocupação de verificar, na prática, a aplicabilidade e reprodutibilidade dos achados obtidos nas pesquisas em laboratório. Assim, desenvolvemos, junto ao Programa de Condicionamento Físico Aplicado à Prevenção Cardiológica Primária e Secundária da EEFEUSP e InCor, uma série de estudos para verificar os efeitos do treinamento físico numa situação real de trabalho. Os dados parciais desses estudos (Alonso, Amaral, Garcia, Campos, Augusto, Alves, Forjaz, Barretto \& Negrão, 1999) têm demonstrado que, mesmo em situações menos controladas, 0 treinamento físico reduz significantemente a freqüência cardíaca de repouso e diminui os níveis pressóricos dos indivíduos que apresentam csses níveis mais elevados.

\section{CONCLUSÃO}

A partir do exposto, fica clara a preocupação do Laboratório de Fisiologia da Atividade Motora em compreender, da forma mais profunda possível, a relação do exercício físico com a função cardiovascular, usando para essa compreensão a realização de pesquisas básicas e aplicadas, em seres humanos e animais de experimentação, de modo a complementar os achados, levando à formação de um corpo de conhecimentos mais amplo. Fica evidente, ainda, que muitas dúvidas precisam ser solucionadas e novos conhecimentos precisam ser adquiridos em futuras investigações do nosso grupo.

\section{REFERÊNCIAS BIBLIOGRÁFICAS}

ALONSO, D.O.; AMARAL, S.L.; GARCIA, M.B.B.; CAMPOS, M.R.; AUGUSTO, J.C.; ALVES, M.J.N.N.; FORJAZ, C.L.M.; BARRETTO, A.C.P.; NEGRÃO, C.E. Efeito de um programa de condicionamento fisico para hipertensos. In: CONGRESSO DA SOCIEDADE BRASILEIRA DE HIPERTENSÃO, 8., Belo Horizonte, 1999.

Resumo das Comunicações Científicas. Belo Horizonte, Sociedade Brasileira de Hipertensão, 1999. p.89.

BRASIL. Ministério da Saúde. Doenças crônicodegenerativas: evolução e tendências atuais. Brasilia, Centro de Documentação do Ministério da Saúde, 1988.

COATS, A.J.S.; CONWAY, J.; ISEA, J.E.; PANNARALE, G.; SLEIGHT, P.; SOMERS, V.K. Systemic and forearm vascular resistance changes after upright bicycle exercise in man. Journal of Physiology, v.413, p.289-98, 1989.

FORJAZ, C.L.M.; MATSUDAIRA, Y.; RODRIGUÊS, F.B.; NUNES, N.; NEGRÃO, C.E. Post-exercise changes in blood pressure, heart rate and rate pressure product at different exercise intensities in normotensive humans. Brazilian Journal of Medical and Biological Research, v.31, n. 10, p.1247-55, 1998a.

FORJAZ, C.L.M.; MION JUNIOR, D.; NEGRÃO, C.E. Effect of aerobic training on casual and ambulatory blood pressure in hypertensives. Circulation, v.94, n.8, p.I373, 1.996a.

- Factors determining post-exercise hypotension in hypertensive humans. In: SCIENTIFIC MEETING OF THE INTER-AMERICAN SOCIETY OF HYPERTENSION, 13., Buenos Aires, 1999. Program - Abstracts. Buenos Aires, Inter-American Society of Hypertension/Sociedad Argentina de Hipertensión Arterial, 1999a. p.A15.

The fall in blood pressure following a single bout of endurance exercise is sustained for 24 hours. Hypertension, v.25, n.6, p.1400, 1995.

Programa de treinamento aeróbio produz melhora na aptidão física e adaptações cardiovasculares, mas não altera o perfil metabólica de indivíduos sadios. Arquivos Brasileiros de Cardiologia, v.67, p.43, 1996b. Suplemento 1 . 
FORJAZ, C.L.M.; RAMIRES, P.R.; TINUCCI, T.; ORTEGA, K.C.; SALOMÃO, H.E.H.; IGNÊS E.C.; WAJCHENBERG B.L.; NEGRÃO, C.E.; MION JUNIOR, D. Post-exercise responses of muscle sympathetic nerve activity, and blood flow to hyperinsulinemia in humans. Journal of Applied Physiology, v.87, n.2, p.824-9, $1999 \mathrm{~b}$.

FORJAZ, C.L.M.; SABINO, S.M.; SQUELLA, S.A.F.; PAULO, A.C.; TINUCCI, T.; RONDON, M.U.P.B.; RONDON, E.; NEGRÃO, C.E. Hemodynamic mechanisms of post-exercise hypotension: influence of exercise intensity. Medicine and Science in Sports and Exercise, v.31, n.5, p.S392, 1999 c. Supplement.

FORJAZ, C.L.M.; SANTAELLA, D.F.; REZENDE, L.O.; BARRETTO, A.C.P.; NEGRÃO, C.E. A duração do exercício determina a magnitude e a duração da hipotensão pós-exercício. Arquivos Brasileiros de Cardiologia, v.70, n.2, p.99-104, 1998b.

GAVA, N.S.; VÉRAS-SILVA, A.S.; NEGRÃO, C.E.; KRIEGER, E.M. Low-intensity exercise training attenuates cardiac $\beta$-adrenergic tone during exercise in spontaneously hypertensive rats. Hypertension, v.26, n.6 (pt.2), p.1129-33, 1995.

GIANOLLA, R.M.; MEDEIROS, A.; KALIL, L.M.P.; MATTOS, K.C.; NEGRÃO, C.E.; BRUM, P.C. Resting bradycardia after swimming training is essentially caused by increase in vagal effect. Medicine and Science in Sports and Exercise, v.30, n.5, p.S217, 1998. Supplement.

KENNEY, M.J.; SEALS, D.R. Postexercise hypotension: key features, mechanisms, and clinical significance. Hypertension, v.22, n.5, p.653-64, 1993.

NEGRÃO, C.E.; IRIGOYEN, M.C.; MOREIRA, E.D.; BRUM, P.C.; FREIRE, P.M.; KRIEGER, E.M. Effect of exercise training on RSNA, baroreflex control, and blood pressure responsiveness. American Journal of Physiology, v.265, n.34, p.R365-70, 1993.
NEGRÃO C.E.; MOREIRA, E.D.; BRUM, P.C.; DENADAI, M.L.D.R.; KRIEGER, E.M. Vagal and sympathetic control of heart rate during exercise by sedentary and exercise-trained rats. Brazilian Journal of Medical and Biological Research, v. 25, n. 10, p. $1045-52,1992 a$.

NEGRÃO, C.E.; MOREIRA, E.D.; SANTOS, M.C.L.M.; FARAH, V.M.A.; KRIEGER, E.M. Vagal function impairment after exercise training. Journal of Applied Physiology, v.72, n.5, p.1749$53,1992 b$.

PAFFENBARGER, R.S.; HADE, R.T.; WING, L.; LEE, I.M.; JUNG, D.L.; KAMPERT, J.B. The association of changes in physical activity level and other lifestyle characteristics with mortality among men. New England Journal Medicine, v.328, n.8, p.538-45, 1993.

RONDON, M.U.P.B. Comportamento imediato e prolongado da pressão arterial pós-exercício em idosos normotensos e hipertensos. São Paulo, 1999. 128p. Tese (Doutorado) - Escola de Educação Física e Esporte, Universidade de São Paulo.

SIlVA, G.J.J.; BRUM, P.C.; NEGRÃO, C.E.; KRIEGER, E.M. Acute and chronic effects of exercise in spontaneously hypertensive rats. Hypertension, v.30, n.3(pt.2), p.714-9, 1997.

VÉRAS-SILVA, A.S.; MATTOS, K.C.; GAVA, N.S.; BRUM, P.C.; NEGRÃO, C.E.; KRIEGER, E.M. Low-intensity exercise training decreases cardiac output and hypertension in spontaneously hypertensive rats. American Journal of Physiology, v.273, n.42, p.H2627-31, 1997.

VÉRAS-SILVA, A.S.; MATTOS, K.C.; IDA, F.; NEGRÃO, C.E.; KRIEGER, E.M. Post-exercise hypotension in spontaneously hypertensive rats is produced by a decrease in cardiac output. Hypertension, v.25, p.I375, 1995.

\author{
ENDEREÇO: Carlos Eduardo Negrão \\ EEFEUSP \\ Av. Prof. Mello Moraes, 65 \\ 05508-900 - São Paulo - SP BRASIL \\ e-mail cenegrao@usp.br
}

